\title{
Review \\ Gut-thyroid axis and celiac disease
}

\author{
Aaron Lerner ${ }^{1,2}$, Patricia Jeremias ${ }^{2}$ and Torsten Matthias ${ }^{2}$ \\ ${ }^{1}$ B. Rappaport School of Medicine, Technion-Israel Institute of Technology, Haifa, Israel \\ ${ }^{2}$ AESKU.KIPP Institute, Wendelsheim, Germany
}

Correspondence

should be addressed

to A Lerner

Email

aaronlerner1948@gmail.com

\begin{abstract}
Autoimmune thyroiditis has an increased prevalence in patients with celiac disease and vice versa. The objective of the current review is to highlight the epidemiological, clinical, serological, pathological, pathophysiological, hormonal, genetic and immunological factors shared between the two entities. They might represent the two ends of the gut-thyroid axis where the cross-talks' pathways are still unravelled. New observations are reviewed, highlighting some gut-thyroid interrelated pathways that potentially might lead to new therapeutic strategies.
\end{abstract}

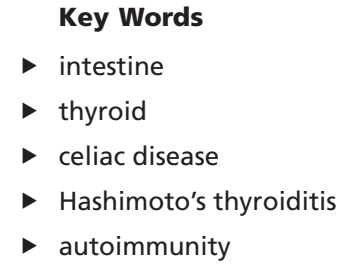

Endocrine Connections (2017) 6, R52-R58

\section{Introduction}

\section{Hashimoto's thyroiditis}

Hashimoto's thyroiditis (HT) is one of the most common autoimmune endocrine diseases, characterized by an autoimmune-mediated destruction of the thyroid gland. Initially considered a rarity, HT has now become the most common autoimmune disease. Its prevalence is $0.8 \%$ when estimated from a review of published articles, $4.6 \%$ when estimated biochemically from the National Health and Nutrition Examination Survey and $>5 \%$ when estimated from cytology of ultrasound-guided fine-needle aspirates of thyroid nodules. Similar to majority of the other autoimmune diseases, HT is age dependent and is more common in women (F:M ratio >10:1) (1). It is identified by anti-thyroglobulin (TG) and/or anti-thyroid peroxidase antibodies (TPO). The disease is a common cause of low thyroid hormones with a high thyroidstimulating hormone (TSH) but some exhibit subclinical hypothyroidism. Interestingly, symptoms of HT and celiac disease (CD) often overlap (Table 1), and both share environmental, pathological, immunogenic, hormonal, serological and genetic aspects $(2,3,4)$. (c) 2017 The authors

http://www.endocrineconnections.org DOI: 10.1530/EC-17-0021

\section{Celiac disease}

$\mathrm{CD}$ is an autoimmune inflammatory disorder of the small intestine, triggered by the ingestion of prolamins contained in wheat, barley or rye, in genetically susceptible individuals. Its incidence in the general population is $1-1.5 \%$. It is gluten depended, thus early diagnosis and subsequent adherence to a gluten-free diet is highly recommended $(5,6)$.

Pathophysiologically, the enzyme tissue transglutamimnase (tTg) is the autoantigen. By posttranslational modification of the absorbed gliadin peptide, deamidating or crosslinking, those peptides are becoming immunogenic/toxic, resulting in mucosal inflammation and damage. Several well established serological markers are available for the diagnosis and follow up: anti-endomysium, anti-deamidated gliadin and anti-tTg autoantibodies. However, two novel ones, the anti-neo-epitope $\mathrm{tTg}$ and the anti-neo-epitope microbial $\mathrm{Tg}$ were recently described with good performances (7). The present review will concentrate on CD and HT, as an example of the gut-thyroid axis. 
Table 1 Shared clinical features between celiac and autoimmune thyroid diseases.

Symptom/sign
Weight
Bowel movement
Joint/bone pain
Fatigue/tiredness
Psychology
Hair loss
Infertility/missed periods
Miscarriage
Increased other autoimmune diseases

\begin{tabular}{l} 
Celiac disease \\
\hline Loss \\
Diarrhea/constipation \\
$+/+$ \\
+ \\
Depression, anxiety
\end{tabular}

\begin{tabular}{l} 
Hashimoto's thyroiditis \\
\hline Gain \\
Constipation \\
+ /-, hypotonia \\
+ \\
Depression
\end{tabular}

\section{Thyroid pathology in celiac disease}

$\mathrm{CD}$ is associated with a number of autoimmune conditions, including HT. The prevalence of autoimmune thyroid disease (ATD) in patients with CD was suggested to be four times higher than that in the general population, though the range is very wide, spanning $1.2-30 \%$. The range for HT in CD is narrower: $1.25-19 \%$ (8). Going vice versa, a Dutch study reported $21 \%$ of the patients with $\mathrm{CD}$ to have HT (8). As ATD is age depended, in the adult populations with $\mathrm{CD}$ the rate of hypothyroidism and/or HT is much higher than that in children, ranging between 12.9 and 30.5\%. Symptoms of undiagnosed CD may be different in patients who also have thyroid disease. Recent studies have shown that it may be beneficial to screen ATD for CD as well $(8,9)$.

\section{Intestinal pathology in thyroid diseases}

Only a single layer of epithelial cells separates the luminal contents from effector immune cells in the lamina propria and the internal milieu of the body. Breaching the epithelium can lead to pathological exposure of the highly immunoreactive sub-epithelium to the vast number of foreign antigens in the lumen, thus driving autoimmunogenesis (10). Increased interest on the thyroid-intestinal epithelium is supplied by the finding of $40 \%$ of patients with HT with lymphocytic colitis, higher intraepithelial lymphocyte counts, dilated tight junctions, and shorter and thicker microvilli. The gastrointestinal dysfunctions in thyroid disorders were most recently reviewed (11).

Taken together, a pathogenic role of the intestinal damage, including in $\mathrm{CD}$, is suggested in the development of HT $(12,13)$. Several potential mechanisms were suggested for the gut mucosa and luminal ecosystems involvement in the thyroid autoimmunity: (i) Gut dysbiosis might disturb the finely tuned immune balance and break tolerance to self-antigens and non-pathogenic non-self-antigens, by posttranslational modification proteins, inducing autoimmunity; (ii) association of lipopolysaccharide-induced Toll-like receptor (TLR) activation with thyroiditis development or production of anti-thyroglobulin antibody in mice was suggested; (iii) induction of Th1 to Th2 shift, inhibition of Th17 differentiation and oral tolerance induction, by retinoic acid might activate tolerogenic immune responses in the gut; (iv) breaching tight junction integrity, resulting in the leaky gut barrier is a common shared pathway in autoimmunogenesis; (v) not less important is the transcriptomic, proteinomic and the metabolomics changes induced by the gut microbes, being direct messengers between the bugs and us. Changes in microbiota and short chain fatty acids production are clearly related to the pathogenesis of $\mathrm{CD}$, but their role in thyroid autoimmunity induction or protection remains to be investigated $(9,10,12,13,14,15,16)$.

\section{Shared autoantibodies}

High positivity of anti-tTgs antibodies among subjects with ATD is well documented. It is recommended to have high clinical index of suspicion for $\mathrm{CD}$ in patients with AT. Higher percentage of anti-tTgs was described in several countries of Asia and Europe.

The opposite picture exists when anti-TPO/anti-TG antibodies are checked in $\mathrm{CD}$. In a recent study, antithyroid antibodies became positive in $16.4 \%$ of the patients 2-3 years after the diagnosis of $\mathrm{CD}$, once again alluding to the age dependence of the ATD.

\section{Shared genes}

Common genetic predisposition has been proposed as a candidate explanation for the positive association
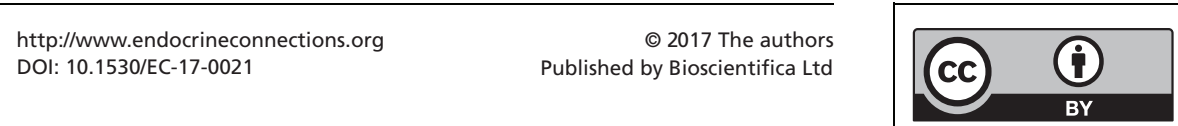
between CD and ATDs. The putative Graves' disease and HT susceptibility genes include both immune modifying genes and thyroid specific genes. The genes that predispose to endocrine autoimmune diseases, i.e. DR3-DQ2 and DR4-DQ8, are also the major genetic determinants of CD, which is the best understood human leukocyte antigen (HLA)-linked disease (17).

The immune-regulatory genes that predispose to autoimmune thyroid diseases (FOXP3, CD25, CD40, CTLA-4, the HLA genes, PTPN22) play critical parts in the development of an effective immune response including self-tolerance. More specifically, CD 40 is shared by Graves' disease and inflammatory bowel disease (IBD), CTLA-4 is shared by Graves' disease, HD, IBD and CD, HLA genes are shared by Graves' disease, HT and CD, and finally PTPN22 is shared by CD, Graves' disease, HT and IBD, thus enhancing the genetic cross-talks in the gut-thyroid axis (18).

\section{Shared immune pathways}

Both diseases, share many immune-pathological pathways: ongoing chronic destructive inflammation and mononuclear infiltration of the target organs, predominance of $\mathrm{T}$ helper cell type 1 pattern response including the associated cytokines such as IL-18 and INF- $\gamma$. Notably, a shared plot was suggested between the two diseases and a new therapeutic strategy, namely, neutralizing antibodies against IL-18 and/or INF- $\gamma$ were suggested as a fruitful option (2).

\section{Shared autoimmune diseases}

Several autoimmune diseases are shared between CD and HT. CD is associated with numerous autoimmune diseases including HT. In a most recent review, multiple diseases were significantly associated with ATD, including CD (19).

\section{Microbiota/dysbiota/metabolome}

The intestinal microbiota can be considered as an endocrine network. Although neglected by the endocrinologists for decades, gut microbiota microbiome represent an important endocrine organ that converts nutritional messages from the intestinal lumen into endocrine signals, impacting the metabolism of local as well as remote organs. Following are several examples of the nutrients-microbiotic-hormonal metabolic axes.

Several observations strengthen the endocrine impact of the microbiota. Hypo responsiveness toward epinephrine, norepinephrine and vasopressin during iodine deprivation, was observed in germ-free rodents. The intestinal microbes can regulate cholesterol, lipid and glucose metabolisms by their bile acid hydrolases capacity, resulting in hormonal release. One of the major metabolic products of the gut microbiome is short-chain fatty acids resulting in numerous luminal and systemic functions (15) affectingleptin, glucagon-likepeptide 1 , ghrelin and peptide YY and productions. The bug's $\beta$-glucuronidases activate norepinephrine and dopamine. Their decarboxylation capacities induce $\gamma$-aminobutyric acid (GABA), tyramine and $\beta$-phenylethylamine productions. Finally, the plasma concentration of tryptophan is dependent on microbiome composition. Being an essential amino acid and precursor of serotonin, tryptophan impact enteric neurotransmitters balance (14).

Interestingly, microbiome can affect the crosstalks between the hypothalamic-pituitary-adrenal axis and behavior. In the opposite aspect, the endogenous hormones can modulate the bacterial proliferative capacity and pathogenicity. Dopamine, norepinephrine, nitric oxide and the inhibitory transmitter GABA are molecules originated from the luminal microbes that influence our endogenous endocrine network (referred to as 'Microbial endocrinology' (14)). The microbial intestinal-thyroiditis interrelations were not described in CD and IBD. Not surprising, probiotics were suggested as a potential therapy for IBD and HT. It can be summarized that in the gut lumen, this type of interrelationship between the bugs and us, is performed continually through various kinds of luminal messengers, part of the luminal metabolome and proteinome, which exert hormonal functions.

Multiple studies support the role of the intestinal commensal microbiota in autoimmunogenesis, including $\mathrm{CD}(10,16)$. In contrast, the role of the intestinal indigenous microorganisms in HT has received little attention. Innate pattern recognition receptors such as TLRs stimulation and breached epithelial tight junction integrity were suggested to contribute to thyroid autoimmunity. Some literature data have suggested that dysbiosis could affect thyroid hormone synthesis and metabolism (20), and it had been suggested that gut bacteria could even deiodinate thyroid hormones, thus affecting serum levels of these hormones (21).

The gut is the first and the widest area of bacteria access, with the highest concentration of T-cells in the human body and trained to react to microorganisms. Interestingly, all the environmental factors involved in the pathogenesis of $\mathrm{CD}$, HT and Graves' disease can alter the balance within the microorganisms located in the 
Table 2 Comparison of various features between celiac and Hashimoto thyroiditis.

\begin{tabular}{|c|c|c|}
\hline & Celiac disease & Hashimoto thyroiditis \\
\hline Incidence & $1-1.5 \%$, increases & $5 \%$, increases \\
\hline Gender predominance & Female & Female \\
\hline Geoepidemiology & Increasing incidence & Increasing incidence \\
\hline Environmental factors & $\begin{array}{l}\text { Gluten, microbial } \mathrm{mTg} \text {, infection, stress, formula } \\
\text { feeding, increased diversity of dysbiota }\end{array}$ & $\begin{array}{l}\text { Infection, diet, iodine, medications, } \\
\text { smoking }\end{array}$ \\
\hline Associated infections & $\begin{array}{l}\text { Enterovirus, EBV, CMV, HBV, HCV, rotavirus } \\
\text { Bacteroides species, Campylobacter jejuni, } \\
\text { pneumococcus, tuberculosis and Helicobacter } \\
\text { pylori }\end{array}$ & $\begin{array}{l}\text { EBV, Yersinia enterocolitica, Helicobacter } \\
\text { pylori, HCV, CMV, Borrelia burgdorferi }\end{array}$ \\
\hline Dysbiota & Decreased diversity & $?$ \\
\hline HLA predisposition & DQ-2, DQ-8 & HLA-DR $\beta 1-A r g 74$, DQ-2 \\
\hline Autoantibodies & $\begin{array}{l}\text { tTg, DGP, EMA, neo-epitope tTg, neo-epitope } \\
\text { mTg }\end{array}$ & Anti-thyroid peroxidase, anti-thyroglobulin \\
\hline Autoantigen & $\mathrm{tTg}$ & Thyroid peroxidase, thyroglobulin \\
\hline Potential inducer enzyme (PTMP) & tTg, mTg deamidation/cross-linking & TTg \\
\hline Adaptive/innate immunity & +++ & +++ \\
\hline Target/associated organs & $\begin{array}{l}\text { Small bowel/joint, bone, endocrine, heart, lung, } \\
\text { liver, kidney, skin, nerves, etc. }\end{array}$ & Thyroid \\
\hline Therapy & Gluten free diet & Symptomatic, thyroid replacement therapy \\
\hline
\end{tabular}

CMV, cytomegalo virus; DGP, diamidated gliadin peptide; EBV, Epshtein Bar virus; EMA, endomysial antibodies; HBV, hepatitis B virus; HCV, hepatitis C virus; $\mathrm{mTg}$, microbial transglutaminase; $\mathrm{tTg}$, tissue transglutaminase.

gut, and influence the immune system, in particular the proportions of regulatory Treg and inflammatory TH17 cells $(15,22)$.

However, the link between the intestine and HT was scarcely investigated, resulting in indirect and weak evidence for such a link, at least, till today (12).

\section{Similarities and dissimilarities between Celiac disease and autoimmune thyroiditis}

The two diseases share multiple similarities and dissimilarities. Following are Tables 1 and 2, summarizing the clinical pictures shared or unshared between CD, HT and Graves' disease (Table 1) and comparison of various features between CD and HT (Table 2).

\section{Gluten and autoimmune thyroiditis}

Literature does not support the use of gluten-free diet (GFD) in the routine treatment of ATD. The possible role of gluten in the induction of the anti-thyroid antibodies as well as, in few cases, the consequent organ dysfunction was suggested. Interestingly, thyroid disease was 3-fold higher in CD than that in controls. In most patients who strictly followed a 1-year GFD, there was a normalization of subclinical hypothyroidism, suggesting that in distinct cases, gluten withdrawal may single-handedly reverse the abnormality. It seems that facing thyroid-associated orbitopathy, CD is the only autoimmune disease where complaints and autoantibodies to tTg usually resolve on a GFD. At least one antibody was positive in 10 of 19 untreated celiacs but only in five of 25 gluten-restricted patients. Once again it shows that gluten withdrawal may change thyroid autoimmunity, mainly when associated with CD.

\section{Gut-thyroid interrelated pathways}

\section{Molecular mimicry}

An unexpected cross-reaction of the antibodies with autologous components may occur. This process is in fact at the basis of the progression of autoimmune diseases and is called molecular mimicry. In the gut, inflammatory pathologies that are related to dysbiosis associated with various factors, such as genetic factors and food, cause alterations of the immune system characterized in IBD and $\mathrm{CD}(9,10,14)$. Those enteric eco-events induce systemic inflammatory responses, leading to the systemic manifestations of IBD and/or CD, affecting remote organs including the thyroid $(10,14)$.

\section{Gut luminal eco-events that might drive gut-thyroid axes}

Dysbiosis The balanced interaction between the host and microbes has been shaped during the long co-evolutionary process. In dysbiotic conditions, this balance is compromised and results in abnormal 

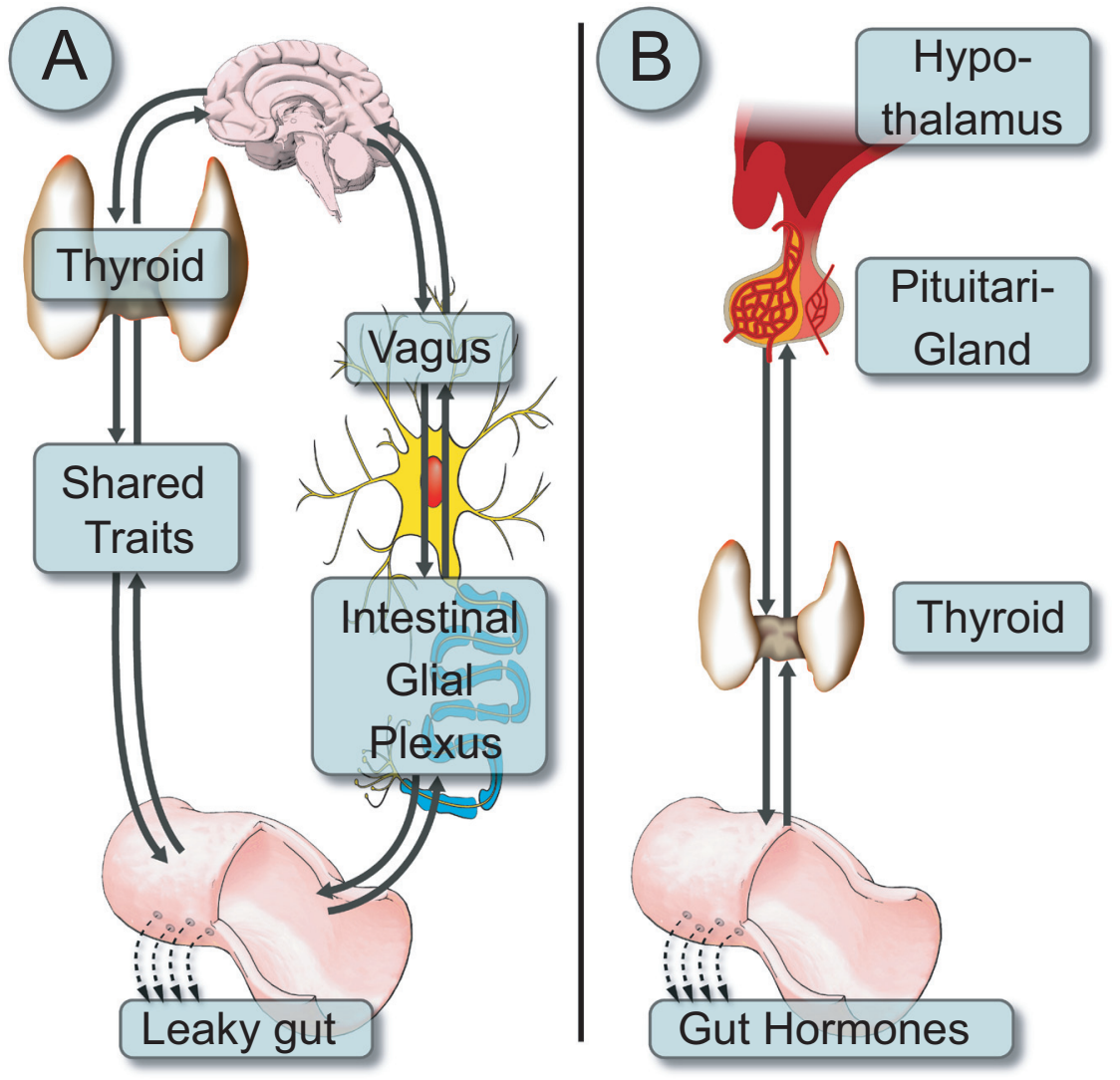

Figure 1

A schematic presentation of $(A)$ the bidirectional neuronal pathways connecting the thyroid through the vagal nerve to the intestinal neuronal plexus, finally inducing leaky gut. Parallel, multiple gut-thyroid shared traits (epidemiology, autoantibodies, genes, immune pathways and autoimmune diseases) influence the gut-thyroid axis; (B) the hormonal bidirectional cross-talks between the hypothalamus-pituitary-thyroid-gut hormones axes. interaction between the host and microbiota (10). In contrast, the place of the altered microbiome, mainly decrease diversity, in autoimmunity induction was well described $(9,10,14)$. Adding the substantial involvement of the microbiome in IBD and CD development and the potential place in HT evolvement, dysbiosis is suggested as a major player in intestinal and thyroidal autoimmunity interaction $(12,13)$.

\section{Posttranslational modification of proteins} (PTMP) PTMP contribute substantially to the adaptability and bacterial cell cycle regulation. Their enzymatic apparatus is capable to transform naïve/self- or non-selfpeptides to autoimmunogenic ones (10). In this regard, CD is a prototype of PTMP by the endogenous tTg and potentially by the microbial $\operatorname{Tg}(9,10,14)$. Further support for the gut-thyroid axis in the recent report of anti-tTg autoantibodies binding to thyroid follicles and extracellular matrix opens a new window for tTg-induced PTMP involvement in the thyroidal autoimmunity (23).

Leaky gut Numerous autoimmune diseases expose increased intestinal permeability (9). This phenomenon was described in $\operatorname{CD}(9,14)$, IBD and thyroidal dysfunction (24). Finally, as microbes are a major breacher of the tight junction integrity and as dysbiosis is crucial in autoimmunogenesis, the leaky gut is assumed to enhance thyroid dysfunction.

\section{tTg and thyroid tissue dysfunction}

tTg antibodies were described to bind to thyroid follicles and extracellular matrix in patients with $\mathrm{CD}$, thus reinforcing the gut-thyroid relationship $(13,23)$. More so, the anti-tTg titers correlate with TPO antibody titers (23). These findings suggest that celiac-associated autoantibodies could contribute to the development of thyroid dysfunction in CD.

\section{Mucosal stress: the heat shock protein theory}

Heat shock proteins (HSP) are stress proteins that have a role in protection of cellsagainst stress. Increased HSP expression was noticed in the intestine and serological anti-HSP autoimmune response was detected in patients with CD and IBD (25). Hsp60 levels are increased in HT and are immunolocalized in the thyroid tissue. Anti-TG

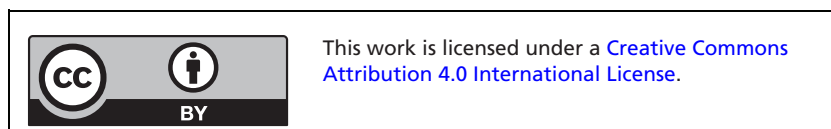


and anti-TPO antibodies cross-react with Hsp60 (26). Those relations need more thorough investigations.

\section{Conclusions}

Despite 104 years of Hashimoto's thyroiditis, and 128 years of CD discovery, both are still intriguing diseases. Multiple epidemiological, clinical, serological, pathological, pathophysiological, genetic, hormonal and immunological aspects are shared between the two (Fig. 1A and B). Increased prevalence of CD-associated antibodies is well described in HT and many recommend screening HT populations for celiac autoimmunity. The routine screening of $\mathrm{CD}$ for thyroid autoimmunity is less established (27). Understanding the cross-talks between the gut eco-system events, the intestinal hormonal repertoire and the thyroid gland (28) might open new therapeutic strategies to combat those diseases.

\section{Declaration of interest}

The authors declare that there is no conflict of interest that could be perceived as prejudicing the impartiality of this review.

\section{References}

1 Guaraldi F, Landek-Salgado MA, Hutfless S, Samoni F, Talor MV, Matos PG, Salvatori R, Rose NR \& Caturegli P. Pituitary antibodies in women with Hashimoto's thyroiditis: prevalence in diagnostic and prediagnostic sera. Thyroid 201222 509-515. (doi:10.1089/ thy.2011.0373)

2 Mormile R. Celiac disease and Hashimoto's thyroiditis: a shared plot? International Journal of Colorectal Disease 201631 947. (doi:10.1007/ s00384-015-2370-z)

3 Zhernakova A, Withoff S \& Wijmenga C. Clinical implications of shared genetics and pathogenesis in autoimmune diseases. Nature Reviews Endocrinology 20139 646-659. (doi:10.1038/nrendo.2013.161)

4 Lerner A \& Matthias T. Autoimmune thyroid diseases in celiac disease: if and when to screen? International Journal of Celiac Disease 20164 124-126.

5 Lerner A, Neidhöfer S \& Matthias T. Serological markers and/or intestinal biopsies in the case-finding of celiac disease. International Journal of Celiac Disease 20153 53-55. (doi:10.12691/ijcd-3-2-7)

6 Lerner A \& Matthias T. Rheumatoid arthritis-celiac disease relationship: joints get that gut feeling. Autoimmunity Reviews 201514 1038-1047. (doi:10.1016/j.autrev.2015.07.007)

7 Matthias T, Jeremias P, Neidhöfer S \& Lerner A. The industrial food additive microbial transglutaminase, mimics the tissue transglutaminase and is immunogenic in celiac disease patients. Autoimmunity Reviews 201615 1111-1119. (doi:10.1016/j. autrev.2016.09.011)

8 Hadithi M, de Boer H, Meijer JW, Willekens F, Kerckhaert JA, Heijmans R, Peña AS, Stehouwer CD \& Mulder CJ. Coeliac disease in Dutch patients with Hashimoto's thyroiditis and vice versa. World Journal of Gastroenterology 200713 1715-1722. (doi:10.3748/wjg.v13. i11.1715)
9 Lerner A \& Matthias T. Changes in intestinal tight junction permeability associated with industrial food additives explain the rising incidence of autoimmune disease. Autoimmunity Reviews 2015 14 479-489. (doi:10.1016/j.autrev.2015.01.009)

10 Lerner A, Aminov R \& Matthias T. Dysbiosis may trigger autoimmune diseases via inappropriate posttranslational modification of host proteins. Frontiers in Microbiology 20167 Article 84. (doi:10.3389/ fmicb.2016.00084)

11 Kyriacou A, McLaughlin J, Akheel A \& Syed AA. Thyroid disorders and gastrointestinal and liver dysfunction: a state of the art review. European Journal of Internal Medicine 201526 563-571. (doi:10.1016/j. ejim.2015.07.017)

12 Mori K, Nakagawa Y \& Ozaki H. Does the gut microbiota trigger Hashimoto's thyroiditis? Discovery Medicine 201214 321-326. (doi:10.3390/microorganisms3020213)

13 Duntas LH. Does celiac disease trigger autoimmune thyroiditis? Nature Reviews Endocrinology 20095 190-191. (doi:10.1038/nrendo.2009.46)

14 Lerner A \& Matthias T. GUT-the Trojan horse in remote organs' autoimmunity. Journal of Clinical and Cellular Immunology 20167401. (doi:10.4172/2155-9899.1000401)

15 Lerner A, Patricia J \& Matthias T. Nutrients, bugs and us: the shortchain fatty acids story in celiac disease. International Journal of Celiac Disease 20164 92-94.

16 Lerner A, Aminov R \& Matthias T. Intestinal dysbiotic transglutaminases are potential environmental drivers of systemic autoimmunogenesis. Frontiers in Microbiology $2017 \mathbf{8}$ article 66.

17 Ban Y \& Tomer Y. Susceptibility genes in thyroid autoimmunity. Clinical and Developmental Immunology 200512 47-58. (doi:10.1080/17402520400008897)

18 Li YR, Li J, Zhao SD, Bradfield JP, Mentch FD, Maggadottir SM, Hou C, Abrams DJ, Chang D, Gao F, et al. Meta-analysis of shared genetic architecture across ten pediatric autoimmune diseases. Nature Medicine 201521 1018-1027. (doi:10.1038/nm.3933)

19 Fallahi P, Ferrari SM, Ruffilli I, Elia G, Biricotti M, Vita R, Benvenga $\mathrm{S} \&$ Antonelli A. The association of other autoimmune diseases in patients with autoimmune thyroiditis: review of the literature and report of a large series of patients. Autoimmunity Reviews 201615 1125-1128. (doi:10.1016/j.autrev.2016.09.009)

20 DiStefano JJ 3rd, de Luze A \& Nguyen TT. Binding and degradation of 3,5,3'-triiodothyronine and thyroxine by rat intestinal bacteria. American Physiological Society 1993264 E966-E972.

21 Fekete C, Gereben B, Doleschall M, Harney JW, Dora JM, Bianco AC, Sarkar S, Liposits Z, Rand W, Emerson C, et al. Lipopolysaccharide induces type 2 iodothyronine deiodinase in the mediobasal hypothalamus: implications for the nonthyroidal illness syndrome. Endocrinology 2004145 1649-1655. (doi:10.1210/ en.2003-1439)

22 Covelli D \& Ludgate M. The thyroid, the eyes and the gut: a possible connection. Journal of Endocrinological Investigation 2017. (doi:10.1007/s40618-016-0594-6)

23 Naiyer AJ, Shah J, Hernandez L, Kim SY, Ciaccio EJ, Cheng J, Manavalan S, Bhagat G \& Green PH. Tissue transglutaminase antibodies in individuals with celiac disease bind to thyroid follicles and extracellular matrix and may contribute to thyroid dysfunction. Thyroid 200818 1171-1178. (doi:10.1089/ thy.2008.0110)

24 Cereijido M, Contreras RG, Flores-Benítez D, Flores-Maldonado C, Larre I, Ruiz A \& Shoshani L. New diseases derived or associated with the tight junction. Archives of Medical Research 200738 465-478. (doi:10.1016/j.arcmed.2007.02.003)

25 Tukaj S, Görög A, Kleszczyński K, Zillikens D, Kárpáti S \& Kasperkiewicz M. Autoimmunity to heat shock proteins and vitamin $\mathrm{D}$ status in patients with celiac disease without associated dermatitis herpetiformis. Journal of Steroid Biochemistry and Molecular Biology 2016 [in press]. (doi:10.1016/j.jsbmb.2016.10.002)

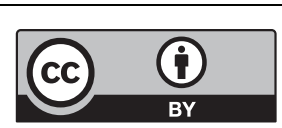


26 Marino Gammazza A, Rizzo M, Citarrella R, Rappa F, Campanella C, Bucchieri F, Patti A, Nikolic D, Cabibi D, Amico G, et al. Elevated blood Hsp60, its structural similarities and cross-reactivity with thyroid molecules, and its presence on the plasma membrane of oncocytes point to the chaperonin as an immunopathogenic factor in Hashimoto's thyroiditis. Cell Stress Chaperones 201419 343-353. (doi:10.1007/s12192-013-0460-9)
27 Reilly NR \& Verma R. Time to screen children with celiac disease for thyroid disease? Journal of Pediatrics $2016 \mathbf{1 7 4}$ 7-9. (doi:10.1016/j. ipeds.2016.04.019)

28 Varian BJ, Poutahidis T, Levkovich T, Ibrahim YM, Lakritz JR, Chatzigiagkos A, Scherer-Hoock A, Alm EJ \& Erdman SE. Beneficial bacteria stimulate youthful thyroid gland activity. Journal of Obesity and Weight Loss Therapy 20144220.

Received in final form 5 April 2017

Accepted 5 April 2017

Accepted Preprint published online 5 April 2017 (c) 2017 The authors Published by Bioscientifica Ltd

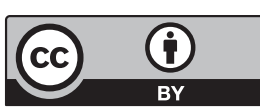

This work is licensed under a Creative Commons Attribution 4.0 International License. 